\title{
A logical analysis of the generalized Banach contractions principle
}

\author{
Alexander P. KReuzer
}

\begin{abstract}
Let $(\mathcal{X}, d)$ be a complete metric space, $m \in \mathbb{N} \backslash\{0\}$, and $\gamma \in \mathbb{R}$ with $0 \leq \gamma<1$. A g-contraction is a mapping $T: \mathcal{X} \longrightarrow \mathcal{X}$ such that for all $x, y \in \mathcal{X}$ there is an $i \in[1, m]$ with $d\left(T^{i} x, T^{i} y\right)<\mathbb{R} \gamma^{i} d(x, y)$.

The generalized Banach contractions principle states that each g-contraction has a fixed point. We show that this principle is a consequence of Ramsey's theorem for pairs over, roughly, $\mathrm{RCA}_{0}+\Sigma_{2}^{0}$-IA.

2010 Mathematics Subject Classification 03B30, 03F60, 47H10 (primary); 03F 35 (secondary)

Keywords: reverse mathematics, Ramsey's theorem for pairs, Banach contraction principle
\end{abstract}

In this paper we will show that a generalization of the Banach contraction mapping principle - the generalized Banach contractions principle - follows from Ramsey's theorem for pairs over a weak basis theory.

Let $(\mathcal{X}, d)$ be a complete metric space, let $m \in \mathbb{N} \backslash\{0\}$, and let $\gamma \in \mathbb{R}$ with $0 \leq \gamma<1$. We call a function $T: \mathcal{X} \longrightarrow \mathcal{X}$ a $(m, \gamma)$-g-contraction if for all $x, y \in \mathcal{X}$ there is an $i \in[1, m]$, such that $d\left(T^{i} x, T^{i} y\right)<\mathbb{R} \gamma^{i} d(x, y)$.

The ordinary Banach contraction mapping theorem states that every $(1, \gamma)$-g-contraction has a fixed point. The generalized Banach contraction mapping principle is the statement that every $(m, \gamma)$-g-contraction has a fixed point.

First results on the generalized Banach contraction mapping principle have been established in Jachymski, Schroder and Stein [12], where it is shown that this principle is true for g-contractions where $m=2$. In Jachymski and Stein [13] it was shown that the principle is true for all $m$ if the g-contraction is uniformly continuous. Later in Merryfield, Rothschild and Stein [18] it was shown that this principle is true for all continuous g-contractions and for $m=3$ without this continuity assumption. The proof of the former statement uses Ramsey's theorem. However, it also uses full arithmetical comprehension, which is - as we will see below - much stronger than this contraction principle. Therefore, 
this proof is not suitable for a faithful formalization. The principle in its full generality was finally proved in Arvanitakis [1] and independently in Merryfield and Stein [19].

\section{Logical Systems}

We will work in the second-order system $R C A_{0}$ and its extension to all finite types $\mathrm{RCA}_{0}^{\omega}$. This use of $\mathrm{RCA}_{0}^{\omega}$ is necessary since the operator $T$ might be non-continuous and has to be presented by a type 2 object in general. (In [12] it was shown that there exists a $(3, \gamma)$-g-contraction which is not continuous.)

The set of all finite types $\mathbf{T}$ is defined to be the smallest set that satisfies

$$
0 \in \mathbf{T}, \quad \rho, \tau \in \mathbf{T} \Rightarrow \tau(\rho) \in \mathbf{T} .
$$

The type 0 denotes the type of natural numbers and the type $\tau(\rho)$ denotes the type of functions from $\rho$ to $\tau$. The type $0(0)$ is abbreviated by 1 the type $0(0(0))$ by 2 . The type of a variable will sometimes be written as superscript of a term.

Equality $={ }_{0}$ for type 0 objects will be added as a primitive notion to the systems together with the usual equality axioms. Higher type equality $=_{\tau \rho}$ will be treated as abbreviation:

$$
x^{\tau \rho}={ }_{\tau \rho} y^{\tau \rho}: \equiv \forall z^{\rho} x z={ }_{\tau} y z .
$$

Define the recursor $R_{\rho}$ of type $\rho$ to be the functional satisfying

$$
R_{\rho} 0 y z={ }_{\rho} y, \quad R_{\rho}\left(S x^{0}\right) y z={ }_{\rho} z\left(R_{\rho} x y z\right) x .
$$

Let Gödel's system $T$ be the $\mathbf{T}$-sorted set of closed terms that can be build up from $0^{0}$, the successor function $S^{1}, \lambda$-abstraction, and the recursors $R_{\rho}$ for all finite types $\rho$. Denote by $T_{0}$ the subsystem of Gödel's system $T$ where primitive recursion is restricted to recursors $R_{0}$. The system $T_{0}$ corresponds to the extension of Kleene's primitive recursive functionals to mixed types whereas full system $T$ corresponds to Gödel's primitive recursive functionals, see $[14,10]$ and [16].

The system $\mathrm{RCA}_{0}^{\omega}$ is defined to be the extension of the term system $T_{0}$ by $\Sigma_{1}^{0}$-induction, the extensionality axioms

$$
\left(\mathrm{E}_{\rho, \tau}\right): \forall z^{\tau \rho}, x^{\rho}, y^{\rho}\left(x={ }_{\rho} y \rightarrow z x={ }_{\tau} z y\right)
$$


for all $\tau, \rho \in \mathbf{T}$, and the schema of quantifier free choice restricted to choice of numbers over functions $\left(\mathrm{QF}_{-} \mathrm{AC} \mathrm{C}^{1,0}\right)$, i.e.

$$
\forall f^{1} \exists x^{0} \mathrm{~A}_{q f}(f, x) \rightarrow \exists F^{2} \forall f^{1} \mathrm{~A}_{q f}(f, F(f)) .
$$

This schema is the higher order equivalent to recursive comprehension $\left(\Delta_{1}^{0}-\mathrm{CA}\right)$. (Strictly speaking the system $\mathrm{RCA}_{0}^{\omega}$ was defined in [15] to contain only quantifier free induction instead of $\Sigma_{1}^{0}$-induction. Since $\Sigma_{1}^{0}$-induction is provable in that system, we may also add it directly.)

It is clear the $R C A_{0}$ can be embedded into RCA $A_{0}^{\omega}$. The system $R C A_{0}^{\omega}$ is conservative over its second-order counterpart, where the second-order part is given by functions instead of sets. This second-order system can then be interpreted in $\mathrm{RCA}_{0}$. See [15].

A complete separable metric space $(\hat{\mathcal{X}}, \hat{d})$ is represented as completion of a countable metric space $(\mathcal{X}, d)$. A point in $\hat{\mathcal{X}}$ is given by a Cauchy sequence of elements of $\mathcal{X}$ having a fixed Cauchy-rate. Thus, a point in $\hat{\mathcal{X}}$ is represented by a type 1 object. The metric $\hat{d}$ is the continuous extension of $d$ to $\hat{\mathcal{X}}$. Two points $x, y \in \hat{\mathcal{X}}$ are defined to be equal $(x=\hat{\mathcal{X}} y)$ if $\hat{d}(x, y)={ }_{\mathbb{R}} 0$. A function $T: \hat{\mathcal{X}} \longrightarrow \hat{\mathcal{X}}$ can then be represented by a type 2 object. To build the iteration $T^{n}$ of $T$ we, in general, require the recursor $R_{1}$, we will therefore work over $\mathrm{RCA}_{0}^{\omega}+\left(R_{1}\right)$, where $\left(R_{1}\right)$ is the axiom that states that the recursor $R_{1}$ exists. See [16, Chapter 4]. Note that over RCA $\mathrm{R}_{0}^{\omega}$ the axiom $\left(R_{1}\right)$ implies $\Sigma_{2}^{0}$-IA and that the provably recursive functions of $\mathrm{RCA}_{0}^{\omega}+\left(R_{1}\right)$ are that same as for $\Sigma_{2}^{0} \mathrm{IA}$, see $[21]$.

In case the function $T$ is continuous in the sense of reverse mathematics, i.e. $T$ has a continuous modulus of continuity, then $T$ can be represented by a type 1 object (or a set), see [23]. One can prove the totality of the iteration $T^{n}$ in $\Sigma_{2}^{0}$-IA and, in fact, it is equivalent to $\Sigma_{2}^{0}$ IA, see [8, Theorem 4.3]. Thus, if one is only interested in such $T$ one could weaken the base theory to $\mathrm{RCA}_{0}+\Sigma_{2}^{0}$-IA. (If one additionally assumes that $\hat{\mathcal{X}}$ is compact then one could also use WKL instead of $\Sigma_{2}^{0}$-IA, see [8, Theorem 4.5].)

We are now in the position to define the generalized Banach contraction mapping principle formally. This definition is relative to $\operatorname{RCA}_{0}^{\omega}+\left(R_{1}\right)$.

Definition 1 Let $\mathrm{GBCC}_{m}$ be that statement that for each presentable complete separable metric space space $(\mathcal{X}, d)$ and each function $T: \mathcal{X} \longrightarrow \mathcal{X}$, which is a $(m, \gamma)$-g-contraction for a $\gamma$ with $0 \leq \gamma<1$ there exists a fixed point of $T$. 
Further, let $\mathrm{GBCC}: \equiv \forall m \mathrm{GBCC}_{m}$ and let $\mathrm{GBCC}_{m}^{c o n t}, \mathrm{GBCC}^{\text {cont }}$ be the restriction of those principles to continuous functions $T$. (GBCC is an abbreviation for "Generalized Banach contraction conjecture".)

The definition of $\mathrm{GBCC}_{m}^{\text {cont }}, \mathrm{GBCC}^{\text {cont }}$ also makes sense in the weaker system $\mathrm{RCA}_{0}+\Sigma_{2^{-}}^{0} \mathrm{IA}$.

Definition 2 (Ramsey's theorem for pairs) Let $[X]^{2}$ be the set of all unordered pairs of $X$. Ramsey's theorem for pairs and $n$ colors $\left(\mathrm{RT}_{n}^{2}\right)$ is the statement that for each coloring of pairs of $\mathbb{N}$ using $n$ colors $c:[\mathbb{N}]^{2} \longrightarrow[0, n[$ there exists an infinite, homogeneous set $X$, i.e., $X$ is infinite and the restriction of $c$ to $[X]^{2}$ is constant. Ramsey's theorem for pairs and arbitrary many colors $\left(\mathrm{RT}_{<\infty}^{2}\right)$ is defined to be $\forall n \mathrm{RT}_{n}^{2}$.

It is easy to see that for each $n \geq 2$ we have $\mathrm{RCA}_{0} \vdash \mathrm{RT}_{2}^{2} \leftrightarrow \mathrm{RT}_{n}^{2}$. However, $\mathrm{RT}_{<\infty}^{2}$ is stronger than $\mathrm{RT}_{2}^{2}$. Therefore, we can restrict our attention to $\mathrm{RT}_{2}^{2}$ and $\mathrm{RT}_{<\infty}^{2}$. It is known that neither $\mathrm{RT}_{2}^{2}$ nor $\mathrm{RT}_{<\infty}^{2}$ imply arithmetical comprehension, [22]. For more details on the strength of these principles, see $[3,11]$.

We will show the following theorem.

\section{Theorem 3}

(i) $\mathrm{RCA}_{0}+\Sigma_{2}^{0}-\mathrm{IA} \vdash \mathrm{RT}_{2}^{2} \rightarrow \mathrm{GBCC}_{m}^{\text {cont }}$ for each $m$,

(ii) $\mathrm{RCA}_{0}+\Sigma_{2}^{0}$-IA $\vdash \mathrm{RT}_{<\infty}^{2} \rightarrow \mathrm{GBCC}^{\text {cont }}$,

(iii) $\mathrm{RCA}_{0}^{\omega}+\left(R_{1}\right) \vdash \mathrm{RT}_{2}^{2} \rightarrow \mathrm{GBCC}_{m}$ for each $m$,

(iv) $\mathrm{RCA}_{0}^{\omega}+\left(R_{1}\right) \vdash\left(\mathrm{RT}_{<\infty}^{2} \wedge \Sigma_{3}^{0}\right.$-IA $) \rightarrow \mathrm{GBCC}$.

Theorem 3 is established by formalizing the proof of the generalized Banach contraction mapping principle of Fremlin [7] and by using some ideas of the proof of [1].

We will first prove the case where $T$ is continuous and then extend it to the general case. Before we can do this, we provide some facts on Ramsey's theorem for pairs and some combinatorial lemmata.

Journal of Logic 8 Analysis 4:17 (2012) 


\section{Combinatorial lemmata}

A coloring $c:[\mathbb{N}]^{2} \longrightarrow[0, n[$ is called stable if $c(\{x, \cdot\})$ eventually becomes constant. The restriction of Ramsey's theorem for pairs to stable colorings is called stable Ramsey's theorem for pairs and denoted by $\mathrm{SRT}_{2}^{2}$ resp. $\mathrm{SRT}_{<\infty}^{2}$.

A set $X$ is called cohesive for a sequence $\left(R_{i}\right)_{i \in \mathbb{N}}$ of subsets of $\mathbb{N}$ if

$$
\forall i\left(X \subseteq^{*} R_{i} \vee X \subseteq^{*} \overline{R_{i}}\right),
$$

where $X \subseteq^{*} Y: \equiv(X \backslash Y$ is finite $)$.

The cohesive principle $(\mathrm{COH})$ states that for every $\left(R_{i}\right)_{i \in \mathbb{N}}$ an infinite, cohesive set exists. The following proposition shows that $\mathrm{COH}$ is the counterpart to the stable Ramsey's theorem.

\section{Proposition 4 ([3, 4])}

- $\mathrm{RCA}_{0} \vdash \mathrm{RT}_{2}^{2} \leftrightarrow \mathrm{COH} \wedge \mathrm{SRT}_{2}^{2}$

- $\mathrm{RCA}_{0} \vdash \mathrm{RT}_{<\infty}^{2} \leftrightarrow \mathrm{COH} \wedge \mathrm{SRT}_{<\infty}^{2}$

Proposition 5 ([3, Lemmas 7.10, 7.12], [5, 4]) Over $\mathrm{RCA}_{0}$ the principle $\mathrm{SRT}_{2}^{2}$ is equivalent to the statement that for every $\Delta_{2}^{0}$-set $A$ there exists an infinite set $X$ such that $X \subseteq A$ or $X \subseteq \bar{A}$.

The principle $\mathrm{SRT}_{<\infty}^{2}$ is equivalent to the statement that for every finite $\Delta_{2}^{0}$-partition $\left(A_{i}\right)_{i<n}$ of $\mathbb{N}$ there exists an $i<n$ and an infinite set $X$ such that $X \subseteq A_{i}$. (If $n$ is uniformly bounded this principle follows from $\mathrm{SRT}_{2}^{2}$ by induction on the metalevel. ${ }^{1}$ )

\footnotetext{
${ }^{1}$ Here and in the following we call a number in a statement uniformly bound if it is bound from outside of the logical system. In Proposition 5 this means that we have that

$$
\mathrm{RCA}_{0}+\mathrm{SRT}_{<\infty}^{2} \vdash \forall n \quad\left\{\begin{array}{l}
\text { for all partitions of } \Delta_{2}^{0} \text {-sets }\left(A_{i}\right)_{i<n} \\
\text { there exists an infinite } X \text { and an } i \text { with } X \subseteq A_{i}
\end{array}\right.
$$
}

and

for all $n \quad \mathrm{RCA}_{0}+\mathrm{SRT}_{2}^{2} \quad \vdash \forall n^{\prime} \leq n\left\{\begin{array}{l}\text { for all partitions of } \Delta_{2}^{0} \text {-sets }\left(A_{i}\right)_{i<n^{\prime}} \\ \text { there exists an infinite } X \text { and an } i \text { with } X \subseteq A_{i}\end{array}\right.$ 
Remark 6 ( $\mathrm{COH}$ as partial non-principal ultrafilter) Let $\left(R_{i}\right)_{i \in \mathbb{N}}$ be a sequence of sets $R_{i} \subseteq \mathbb{N}$ and let $S$ be an infinite cohesive set for this sequence.

Define $\mathcal{F} \subseteq \mathcal{P}(\mathbb{N})$ by

$$
X \in \mathcal{F} \quad \text { iff } \quad S \subseteq^{*} X .
$$

Then as long as one is only concerned about sets in $\left(R_{i}\right)_{i}$ the usual properties of a non-principal ultrafilter hold; i.e. let $i, j \in \mathbb{N}$ then

- $R_{i} \subseteq R_{j} \wedge R_{i} \in \mathcal{F} \Rightarrow R_{j} \in \mathcal{F}$,

- $R_{i}, R_{j} \in \mathcal{F} \Rightarrow R_{i} \cap R_{j} \in \mathcal{F}$,

- $R_{i} \in \mathcal{F} \vee \overline{R_{i}} \in \mathcal{F}$ (by cohesiveness of $S$ ),

- $R_{i} \in \mathcal{F} \Rightarrow R_{i}$ is infinite.

In other words, $\mathcal{F}$ defines a non-principal ultrafilter in the algebra of sets created by $\left(R_{i}\right)_{i}$. Hence, if one can fix in advance a countable number of sets, for which the properties of a non-principal ultrafilter are needed, the ultrafilter may be replaced by the filter $\mathcal{F}$.

Note that the statement $X \in \mathcal{F}$ is $\Delta_{2}^{0}(S)$ for $X \in\left(R_{i}\right)$.

\subsection{Syndetic sets}

Definition 7 (Syndetic)

- Let $m \geq 1$. A set $I \subseteq \mathbb{N}$ is called $m$-syndetic if for all $k \in \mathbb{N}$ the set $I \cap[k, k+m[$ is not empty.

- A set $I \subseteq \mathbb{N}$ is called piecewise $m$-syndetic if there exists arbitrary large intervals $\left[j_{1}, j_{2}\right]$, such that for all $k \in\left[j_{1}, j_{2}-m\right]$ the set $I \cap[k, k+m[$ is not empty.

Lemma $8 \quad\left(\mathrm{RCA}_{0}+\mathrm{SRT}_{<\infty}^{2}\right)$ Let $n \in \mathbb{N}$. If $\left(A_{i}\right)_{i<n}$ is a finite sequence of pairwise disjoint $\Delta_{2}^{0}$-subsets of $\mathbb{N}$, such that $I:=\bigcup_{i<n} A_{i}$ is $m$-syndetic for an $m$, then there exists an infinite set $X$ such that $X \subseteq A_{i}$ for an $i$.

This lemma requires $\mathrm{SRT}_{2}^{2}$ if $n$ and $m$ are fixed and $\mathrm{SRT}_{<\infty}^{2}$ otherwise.

Proof Define a $\Delta_{2}^{0}$-function $f: \mathbb{N} \longrightarrow[0, n]$, via a $\Delta_{2}^{0}$-formula for its graph, denoting to which set a number belongs, by

$$
f(x):= \begin{cases}i & \text { if } x \in A_{i}, \\ n & \text { otherwise }\end{cases}
$$

Journal of Logic 8 Analysis 4:17 (2012) 
We now divide the natural numbers into blocks of size $m$, and define the $\Delta_{2}^{0}$-function $g$ assigning to each of those blocks the sequence of values of $f$ on it:

$$
g(x):=\langle f(x \cdot m), \ldots, f(x \cdot m+m-1)\rangle
$$

Note that because $I$ is $m$-syndetic $g(x) \neq\langle n, \ldots, n\rangle$ for all $x$. The function $g(x)$ defines a $\Delta_{2}^{0}$-partition $\left(B_{i}\right)_{i<n^{\prime}}$ of $\mathbb{N}$ with

$$
B_{i}:=\{x \mid g(x)=i\}, \quad n^{\prime}:=\langle\underbrace{n, \ldots, n}_{m \text { times }}\rangle .
$$

By Proposition 5 we can find an infinite set $Y$ on which $g$ is constant. Since $g(Y) \neq\langle n, \ldots, n\rangle$ there is a $j<m$ such that $(g(Y))_{j} \neq n$. Let $X:=\{x \cdot m+j \mid$ $x \in Y$ \}. By definition $f$ is constant on $X$ and $f(X) \neq n$. Thus, $X \subseteq A_{f(X)}$.

The original proof of Arvanitakis uses the well known fact that piecewise syndetic is a partition stable property. This was proved by Brown in [2] and others later, see for instance [9, Theorem 1.23]. These proofs use the Bolzano-Weierstraß principle for the Cantor space and hence comprehension and are, therefore, not faithful, i.e. the proofs do not formalize in a system weaker than $\mathrm{ACA}_{0}$. Luckily we only need the following weaker facts about partitions of even syndetic sets and not piecewise syndetic sets.

The following two lemmas are based on [2, Lemma 1].

Lemma $9 \quad\left(\mathrm{RCA}_{0}\right)$ Let $X$ be an $m$-syndetic set. If $X$ is partitioned into 2 parts $A_{0}, A_{1}=X \backslash A_{0}$ then either each $A_{i}$ is piecewise $m$-syndetic or there are $i<2$ and $k$ such that $A_{i}$ is $k$-syndetic.

Proof Suppose that there is no $k$ such that $A_{0}$ is $k$-syndetic. Then there are intervals $I$ of arbitrary length such that $A_{0} \cap I=\emptyset$. This means that $A_{1} \cap I=X \cap I$ hence $A_{1}$ is piecewise $m$-syndetic. Same for $A_{0}$.

Corollary $10\left(\mathrm{RCA}_{0}+\Sigma_{2}^{0}\right.$-IA $)$ Let $X$ be an $m$-syndetic set. If $X$ is partitioned into finitely many parts $\left(A_{i}\right)_{i<n}$ then there is an $J \subseteq[0, n[$ and an $k$ such that each $A_{i}$ with $i \in J$ is piecewise $k$-syndetic and $Y:=\bigcup_{i \in J} A_{i}$ is $k$-syndetic.

If the numbers of partitions $n$ is uniformly bounded no $\Sigma_{2}^{0} \mathrm{IA}$ is needed. 
Proof Note that being $k$-syndetic is a $\Pi_{1}^{0}$-statement $(\forall x \exists y<x+k(y \in X))$. We search for a $\subseteq$-minimal set $J \subseteq\left[0, n\right.$ [, such that there is a $k$ with $\bigcup_{i \in J} A_{i}$ is $k$-syndetic. To do so we build by finite $\Sigma_{2}^{0}$-comprehension a finite sequence $s$ such that

$$
(s)_{j}=0 \quad \text { iff } \quad\left\{\begin{array}{l}
\text { there is a } k, \text { such that if } j \text { codes the set } J \\
\text { then } \bigcup_{i \in J} A_{i} \text { is } k \text {-syndetic }
\end{array}\right.
$$

and then search for a minimal set. This finite comprehension requires $\Sigma_{2}^{0}$-induction if greatest index of a set $J \subseteq[0, n$ [ is not fixed, i.e. if $n$ is not uniformly bounded.

The case $J=\emptyset$ is ruled out because then $\bigcup_{i \in J} A_{i}=\emptyset$ and thus would not be syndetic. Let $j \in J$. Heading for a contradiction suppose that $A_{j}$ is not piecewise $k$-syndetic. Then by Lemma 9 the set $\bigcup_{i \in J \backslash\{j\}} A_{i}$ must be $m$-syndetic for an $m$. Thus, $J$ is not $\subseteq$-minimal with this property which contradicts our choice of $J$.

Combining Lemma 8 and Corollary 10 we obtain the following proposition.

Proposition $11\left(\mathrm{RCA}_{0}+\mathrm{SRT}_{<\infty}^{2}+\Sigma_{3}^{0} \mathrm{IA}\right)$ Let $X$ be an $m$-syndetic set. If $X$ is partitioned into $\Delta_{2}^{0}$-sets $\left(A_{i}\right)_{i<n}$, then there exists an $i$ such that $A_{i}$ is piecewise $k$-syndetic and an infinite set $I$ such that $I \subseteq A_{i}$. Note that we do not require $I$ to be piecewise syndetic.

If $n$ is uniformly bounded only $\mathrm{SRT}_{2}^{2}$ is needed. Otherwise, $\Sigma_{3}^{0}$-IA and $\mathrm{SRT}_{<\infty}^{2}$ is needed.

Proof By Corollary 10 we can find a set $J$ such that $\left(A_{i}\right)_{i \in J}$ is syndetic and each $A_{i}$ with $i \in J$ is piecewise syndetic. Note that $\Sigma_{3}^{0}$-induction is needed since the partition is $\Delta_{2}^{0}$. An application of Lemma 8 now proves the proposition.

\section{The proof of GBCC}

\subsection{The continuous case}

Fix a provably presentable complete separable metric space $(\mathcal{X}, d)$ and a $(m, \gamma)$-g-contraction $T: \mathcal{X} \longrightarrow \mathcal{X}$ which is continuous. 
Lemma $12\left(\mathrm{RCA}_{0}+\Sigma_{2}^{0}\right.$-IA, [7, Lemma 2]) For all points $x, y \in \mathcal{X}$ the set

$$
I:=\left\{i \in \mathbb{N} \mid d\left(T^{i} x, T^{i} y\right)<_{\mathbb{R}} \gamma^{i} d(x, y)\right\}
$$

is m-syndetic.

Proof By the g-contraction property $I \cap[1, m] \neq \emptyset$ and for each $i \in I$ there is a $j \in[1, m]$ such that $i+j \in I$.

Lemma $13\left(\mathrm{RCA}_{0}+\Sigma_{2}^{0}\right.$-IA, [18, Lemma 1], [7, Lemma 5]) For each $x \in \mathcal{X}$ there exists an $M>_{\mathbb{R}} 0$ such that the set

$$
I:=\left\{i \in \mathbb{N} \mid d\left(T^{i} x, x\right)<_{\mathbb{R}} M\right\}
$$

is $m$-syndetic.

Proof Let $M=\frac{2}{1-\gamma} \max _{i \in[0, m]} d\left(T^{i} x, x\right)$. (We assume that $T x \neq \mathcal{X} x$ here, otherwise we would be done.) It is clear that $0 \in I$. For each $i \in I$ there is a $j \in[1, m]$ such that $d\left(T^{j+i} x, T^{j} x\right)<_{\mathbb{R}} \gamma^{j} d\left(T^{i} x, x\right)<_{\mathbb{R}} \gamma M$ and hence

$$
d\left(T^{i+j} x, x\right)<_{\mathbb{R}} \gamma M+d\left(T^{j} x, x\right)<_{\mathbb{R}} \gamma M+(1-\gamma) M=M
$$

and thus $i+j \in I$.

Remark 14 It is clear that the Lemmas 12 and 13 also hold for non-continuous $T$ if the theory $\mathrm{RCA}_{0}+\Sigma_{2}^{0}$-IA is replaced by $\mathrm{RCA}_{0}^{\omega}+\left(R_{1}\right)$.

Lemma $15\left(\mathrm{RCA}_{0}+\Sigma_{3}^{0}-\mathrm{IA}+\mathrm{RT}_{<\infty}^{2},[1,4.2],[7\right.$, Lemma 4])

Let $R \subseteq \mathbb{N} \times \mathbb{N}$ be such that

(i) the set $\{i \mid(i, 0) \in R\}$ is $m$-syndetic,

(ii) for every $(i, j) \in R$ the set $\{k \mid(i+k, j+k) \in R\}$ is $m$-syndetic.

Then there exists an infinite set $I$ and a piecewise syndetic $\Delta_{2}^{0}$-set $\tilde{I}$ such that $I \subseteq \tilde{I} \subseteq \mathbb{N}$ and for every $i, j \in \tilde{I}$ there is a $k$ with

$$
(k, i) \in R \text { and }(k, j) \in R .
$$

If $m$ is fixed then $\mathrm{RT}_{2}^{2}$ suffices. If the existence of an $I$, such that for all $i, j \in I$ there is a $k$ satisfying (1), is sufficient (in other words the $\Delta_{2}^{0}$-set $\tilde{I}$ is not needed) then $\mathrm{RT}_{<\infty}^{2}$ suffices. Otherwise, $\mathrm{RT}_{<\infty}^{2}$ and $\Sigma_{3}^{0}$-IA is needed. 
Proof We claim that $R$ meets $[l, l+2 m[\times[k, k+m[$ for all $k, l \in \mathbb{N}$ with $k \leq l$. To prove this claim note that by (i) there is an $i \in[l-k, l-k+m[$ such that $(i, 0) \in R$, and by (ii) there is now a $j \in[k, k+m[$ such that $(i+j, j) \in R$ and that also $(i+j, j) \in[l, l+2 m[\times[k, k+m[$.

For each $i \in \mathbb{N}$ and $j<2 m$ let $L_{i j}:=\{l \mid(l+j, i) \in R\}$. Using the cohesive principle (which follows from $\mathrm{RT}_{2}^{2}$, see Proposition 4 ) we find a cohesive set $S$ for $\left(L_{i j}\right)_{i, j}$ and a non-principal ultrafilter $\mathcal{F}:=\left\{X \mid S \subseteq^{*} X\right\}$ in the algebra created by $\left(L_{i j}\right)$. The ultrafilter is $\Delta_{2}^{0}$, see Remark 6 .

By the claim it follows that

$$
\bigcup_{\substack{i \in[k, k+m[ \\j<2 m}} L_{i j} \supseteq[k, \infty[.
$$

Hence by the ultrafilter property of $\mathcal{F}$ there is for each $k$ some $i \in[k, k+m[$ and $j<2 m$ such that $L_{i j} \in \mathcal{F}$.

Now for $j<2 m$ define $I_{j}:=\left\{i \mid L_{i j} \in \mathcal{F}\right\}$. Observe that by the previous argument the set $\bigcup_{j<2 m} I_{j}$ is $m$-syndetic. The sets $I_{j}$ are $\Delta_{2}^{0}$-set since $\mathcal{F}$ is.

Using Proposition 11 we can find an infinite set $I$ and a $j$ such that $I_{j}$ is piecewise syndetic and $I \subseteq I_{j}$.

If $i, i^{\prime} \in I_{j}$, then $L_{i j}$ and $L_{i^{\prime} j}$ belong to $\mathcal{F}$, so they cannot be disjoint. Thus, there is some $l$ such that $(l+j, i)$ and $\left(l+j, i^{\prime}\right)$ belong to $R$. Hence, $I$ and $\tilde{I}=I_{j}$ satisfies the lemma. If one is only interested in $I$ then Lemma 8 instead of Proposition 11 suffices.

We are now in the position to show Theorem 3 restricted to the continuous case, i.e.

(i) $\mathrm{RCA}_{0}+\Sigma_{2}^{0}$-IA $\vdash \mathrm{RT}_{2}^{2} \rightarrow \mathrm{GBCC}_{m}^{\text {cont }}$ for each $m$,

(ii) $\mathrm{RCA}_{0}+\Sigma_{2}^{0} \mathrm{IA} \vdash \mathrm{RT}_{<\infty}^{2} \rightarrow \mathrm{GBCC}^{\text {cont }}$.

Proof of Theorem 3 for the continuous case Fix an arbitrary $x \in \mathcal{X}$. By Lemma 13 an $M>_{\mathbb{R}} 0$ exists, such that $\left\{i \mid d\left(T^{i} x, x\right)<_{\mathbb{R}} M\right\}$ is $m$-syndetic. We further may assume that $M>_{\mathbb{R}} d(T x, x)$. Let $R \subseteq \mathbb{N} \times \mathbb{N}$ be the relation

$$
R:=\left\{(i, j) \mid d\left(T^{i} x, T^{j} x\right)<_{\mathbb{R}} M \gamma^{j}\right\}
$$

By definition $\{i \mid(i, 0) \in R\}$ is $m$-syndetic. If $(i, j) \in R$ then

$$
\{k \mid(i+k, j+k) \in R\} \supseteq\left\{k \mid d\left(T^{i+k} x, T^{j+k} x\right)<\mathbb{R} \gamma^{k} d\left(T^{i} x, T^{j} x\right)\right\}
$$


and hence is by Lemma 12 also $m$-syndetic.

The set $R$ satisfies the assumptions of Lemma 15. This lemma is not directly applicable since the set $R$ is just a $\Sigma_{1}^{0}$-set because $<_{\mathbb{R}}$ is a $\Sigma_{1}^{0}$-statement. However, we can easily build a recursive set $R^{\prime} \subseteq R$ satisfying also the assumptions of Lemma 15: By QF-AC ${ }^{0,0}$ and the properties of $R$ we can find a function $f_{1}(i, w)$ such that if $w$ is a witness for $(i, 0) \in R$ then $f_{1}(i, w)=\left(k, w^{\prime}\right)$ with $k<m$ and $w^{\prime}$ witnesses that $(i+k+1,0) \in R$. Similarly there exists a function $f_{2}(i, j, w)=\left(k, w^{\prime}\right)$ for the second property. Now let $w$ be a witness for the fact that $(1,0)$ is in $R$. Let

$$
\begin{aligned}
R_{0}^{\prime}:= & \{(1,0, w)\}, \\
R_{n+1}^{\prime}:= & \left\{\left(i+k+1,0, w^{\prime}\right) \mid(i, 0, w) \in R_{n}^{\prime} \text { and } f_{1}(i, w)=\left(k^{\prime}, w^{\prime}\right)\right\} \\
& \cup\left\{\left(i+k+1, j+k+1, w^{\prime}\right) \mid(i, j, w) \in R_{n}^{\prime} \text { and } f_{2}(i, j, w)=\left(k^{\prime}, w^{\prime}\right)\right\},
\end{aligned}
$$

and let $R^{\prime}$ be the projection of $\bigcup_{n} R_{n}^{\prime}$ to the first two components. The membership in $R^{\prime}$ is decidable, since the first component of the elements of the sets $\left(R_{n}^{\prime}\right)$ always increases and thus $(i, j) \in R^{\prime}$ iff $\exists w(i, j, w) \in \bigcup_{n \leq i} R_{n}^{\prime}$. The $\exists$-quantifier here is decidable since the sets $\left(R_{n}\right)$ are finite. By definition $R^{\prime}$ satisfies the assumptions of Lemma 15 and is a subset of $R$.

Hence there is an infinite set $I \subseteq \mathbb{N}$ such that for all $i, j \in I$ there is a $k \in \mathbb{N}$ such that $(k, i),(k, j) \in R^{\prime} \subseteq R$. By definition of $R$ we have

$$
d\left(T^{i} x, T^{j} x\right) \leq_{\mathbb{R}} d\left(T^{k} x, T^{i} x\right)+d\left(T^{k} x, T^{j} x\right) \leq_{\mathbb{R}} M \gamma^{i}+M \gamma^{j} \underset{i, j \rightarrow \infty}{\longrightarrow} 0 .
$$

Thus, the sequence $\left(T^{i} x\right)_{i \in I}$ is a Cauchy-sequence with Cauchy-rate $2 M \gamma^{i}$ and admits a limit point, call it $z$.

Note that by continuity of $T$ for all $k$ we have

$$
\lim _{i \in I} T^{i+k} x=T^{k} z
$$

Since $(1,0) \in R^{\prime}$, the set $L:=\left\{k \mid(1+k, k) \in R^{\prime}\right\} \subseteq\{k \mid(1+k, k) \in R\}$ is $m$-syndetic and so we can find for every $i \in I$ a $j_{i} \in\left[0, m\left[\right.\right.$ such that $i+j_{i} \in L$, i.e.

$$
d\left(T^{i+j_{i}+1} x, T^{i+j_{i}} x\right) \leq_{\mathbb{R}} M \gamma^{i+j_{i}} .
$$

By the infinite pigeonhole principle there is a $j$ and an infinite set $J \subseteq I$ on which $j_{i}=j$ is constant. For every $i \in J$ then holds

$$
\begin{aligned}
d\left(T^{j} z, T^{j+1} z\right) & \leq d\left(T^{j} z, T^{i+j} x\right)+d\left(T^{i+j} x, T^{i+j+1} x\right)+d\left(T^{i+j+1} x, T^{j+1} z\right) \\
& \leq d\left(T^{j} z, T^{i+j} x\right)+M \gamma^{i+j}+d\left(T^{i+j+1} x, T^{j+1} z\right)
\end{aligned}
$$


The last expression tends to 0 as $i \in J$ tends to infinity. This yields that $T^{j} z$ is a fixed-point.

The proof formalizes in $\mathrm{RCA}_{0}+\Sigma_{2}^{0}$-IA except for Lemma 15, where we need $\mathrm{RT}_{2}^{2}$ is $m$ is uniformly bounded and $\mathrm{RT}_{<\infty}^{2}$ otherwise. Hence, the statement follows.

Remark 16 A careful inspection of the proof show that in the proof of (i) the principle $\Sigma_{2}^{0}$-IA is only used to establish that the all iterates $T^{n}$ of $T$ exists. This is needed to build the set $R$. Thus, $\mathrm{GBCC}_{m}^{c o n t}$ restricted to g-contractions $T$ where $T^{n}$ provably exists follows from $\mathrm{RT}_{2}^{2}$ over $\mathrm{RCA}_{0}$ without $\Sigma_{2}^{0}$-IA.

\subsection{Proof of the general case}

Now let $T: \mathcal{X} \longrightarrow \mathcal{X}$ be an arbitrary g-contraction.

Lemma $17\left(\operatorname{RCA}_{0}^{\omega}+\left(R_{1}\right),[7\right.$, Lemma 3]) Let $x \in \mathcal{X}$. If there exists an $n \geq 1$ such that $T^{n} x=x$ then already $T x=\mathcal{X} x$.

Proof Assume that $n$ is minimal with $T^{n} x=\mathcal{X}$. Since $x=\mathcal{X} y$ is $\Pi_{1}^{0}$ one can find such an $n$ using $\Sigma_{1}^{0}$-IA.

If $n \geq 2$ take $i<j \in\left[1, n\left[\right.\right.$ such that $d\left(T^{i} x, T^{j} x\right)$ is minimal. Again $\Sigma_{1}^{0}$-IA proves that such $i, j$ exists.

By the $(m, \gamma)$-g-contraction property there is a $k \in[1, m]$ such that

$$
\gamma^{k} d\left(T^{i} x, T^{j} x\right)>_{\mathbb{R}} d\left(T^{i+k} x, T^{j+k} x\right) .
$$

By the assumption $T^{n}(x)=x$ the right side is equal to $d\left(T^{(i+k) \bmod n} x, T^{(j+k) \bmod n} x\right)$ which is a contradiction to the minimality.

Hence $n=1$ and $T x=\mathcal{X}$.

Lemma $18\left(\mathrm{RCA}_{0},[1\right.$, Lemma 3.2]) Let $N$ be a given multiple of $m$. Then for all $u, v \in \mathbb{N}$ there exists a number $p(u, v) \in \mathbb{N}$ such that whenever $R \in$ $[1, p(u, v)] \times[0, \infty[$ is a relation satisfying

(1) the set $\{i \mid(i, 0) \in R\}$ meets every sets $[k+1, k+N] \subseteq[1, p(u, v)]$,

(2) if $i+m \leq p(u, v)$ and $(i, j) \in R$, then there are $1 \leq i^{\prime}, j^{\prime} \leq J$ such that $\left(i+i^{\prime}, j+j^{\prime}\right) \in R$, 
then there exists a subinterval $[k+1, k+N] \subseteq[1, p(u, v)]$ and $k_{1}, \ldots, k_{u} \in \mathbb{N}$ such that

(1) $k_{r+1}-k_{r} \geq m$ for $1 \leq r<u$,

(2) for every $k_{r}$ there exists a $q \in[k+1, k+N]$ such that $\left(q, k_{r}\right) \in R$.

Proof The proof of Arvanitakis in [1, Lemma 3.2] uses only quantifier free induction and can be formalized even in elementary arithmetic.

Lemma $19\left(\operatorname{RCA}_{0}^{\omega}+\left(R_{1}\right)\right.$, [1, Lemma 3.1]) Assume that no power of $T$ has a fixed-point, then for every $N \in \mathbb{N}$ there exists a $p(N) \in \mathbb{N}$ such that for every point $z \in X$ there exists an $\varepsilon>_{\mathbb{R}} 0$ with the property that for every $y \in X$ one finds $N$ successive iterates of $T$ in the set $y, T y, \ldots, T^{p(N)-1} y$ whose distance to $z$ is bigger than $\varepsilon$.

Proof This lemma is an elementary application of the previous lemma. The proof of Arvanitakis ([1, Lemma 3.1]) can also be formalized in this system.

Proof of Theorem 3 Like in the continuous case we construct using Lemma 15 an infinite set $I$. We now use that this lemma also provides a piecewise $N$-syndetic $\Delta_{2}^{0}$-set $\tilde{I}$, such that $I \subseteq \tilde{I} \subseteq \mathbb{N}$. Again $\left(T^{i} x\right)_{i \in \tilde{I}}$ is a Cauchy-sequence with Cauchyrate $2 M \gamma^{i}$ and limit point $z$. Note that the sequence restricted to the elements in $I$ converges to $z$, too. Hence, $z$ is definable in the system.

Assume for a contradiction that $T$ has no fixed point. By Lemma 17 no power of $T$ has a fixed point and hence by Lemma 19 for a given $N$ there are $p(N), \varepsilon$, such that for every point $y \in \mathcal{X}$ in $\left(T^{i} y\right)_{i \in[1, p(N)]}$ there are $N$ successive elements, which are more than $\varepsilon$ apart from $z$.

By the convergence of $\left(T^{i} x\right)_{i \in \tilde{I}}$ there exists an $i_{0}$ such that

$$
d\left(T^{i} x, z\right)<\varepsilon \quad \text { for } i \in \tilde{I} \text { and } i \geq i_{0} .
$$

The $\Delta_{2}^{0}$-set $\tilde{I}_{0}:=\tilde{I} \cap\left[i_{0}, \infty[\right.$ is evidently also piecewise $N$-syndetic.

Using the piecewise $N$-syndetic property of $\tilde{I}_{0}$ one can find a subset of size $p(N)$ where at least every $N$-th element is $\varepsilon$-close to $z$, contradicting the conclusion of Lemma 19 and thus the assumption that $T$ has no fixed-point.

This proves the theorem.

Again, the proof formalizes in $\mathrm{RCA}_{0}^{\omega}+\left(R_{1}\right)$ except for Lemma 15, where we need $\mathrm{RT}_{2}^{2}$ is $m$ if uniformly bounded and $\mathrm{RT}_{<\infty}^{2}$ and $\Sigma_{3}^{0}$-IA otherwise. Hence, the statement follows. 


\section{Final remarks}

We showed that the generalized Banach contractions principle follows from Ramsey's theorem for pairs. The proof depends essentially on the fact that Ramsey's theorem splits into stable Ramsey's theorem and the cohesive principle. This shows that this split is not only useful as a technical tool to investigate the strength of $\mathrm{RT}_{2}^{2}$ but also is (implicitly) used outside of logic.

The principle GBCC ${ }^{\text {cont }}$ trivially implies $\Sigma_{2}^{0}$-IA since it assumes by formulation that for each functional $T$ its iterates $T^{n}$ exists which implies - as mentioned in Section $1-\Sigma_{2}^{0}$-induction. For the principle $\mathrm{GBCC}_{m}^{\text {cont }}$ the formulation does not require $\Sigma_{2}^{0}$-induction, since only iterations of $T$ up to the uniform bound $m$ are needed.

We do not know whether $\mathrm{GBCC}_{m}^{\text {cont }}$ or even the restriction mentioned in Remark 16 implies $\Sigma_{2}^{0}$-induction. Given the fact that for $\mathrm{RT}_{2}^{2}$ this question is still open, see [20], and for the weaker principle $\mathrm{SRT}_{2}^{2}$ this was just recently resolved, see [6], this question might be very difficult to answer.

The usual Banach contractions principle is provable in the base system $\mathrm{RCA}_{0}$. Therefore, GBCC ${ }^{\text {cont }}$ is stronger than the usual Banach contractions principle. We were not able to show that $\mathrm{GBCC}_{m}^{\text {cont }}$ does not follow from $\mathrm{RCA}_{0}$. Thus, it might be possible that this principle coincide with the Banach contractions principle. Given that fact that the combinatorics of the generalized Banach contractions principle is much more complicated we conjecture that this is not the case and that there is a computable instance of $\mathrm{GBCC}_{m}^{\text {cont }}$ having no computable solution. However, it remains open to determine the strength of the generalized Banach contractions principle below Ramsey's theorem for pairs.

Our formalization shows that program extraction techniques we developed in [17] are applicable. It is a first step to extract quantitative content-like rates of asymptotic regularity - from the generalized Banach contractions principle.

\section{Acknowledgments}

The author was supported by the German Science Foundation (DFG Project KO 1737/5-1) and the Récré project.

The results of this paper are part of the author's dissertation, written under the supervision of Prof. Ulrich Kohlenbach. 
The author would like to thank the anonymous referees for comments and suggestions that have helped to improve the presentation of this paper.

\section{References}

[1] A D Arvanitakis, A proof of the generalized Banach contraction conjecture, Proc. Amer. Math. Soc. 131 (2003), 3647-3656; doi: 10.1090/S0002-9939-0306937-5.

[2] T C Brown, An interesting combinatorial method in the theory of locally finite semigroups, Pacific J. Math. 36 (1971), 285-289.

[3] P A Cholak, C G Jockusch, Jr, T A Slaman, On the strength of Ramsey's theorem for pairs, J. Symbolic Logic 66 (2001), 1-55; doi: 10.2307/2694910.

[4] P A Cholak, C G Jockusch, Jr, T A Slaman,corrigendum to: On the strength of Ramsey's theorem for pairs, J. Symbolic Logic 74 (2009), 1438-1439; doi: $10.2178 / \mathrm{jsl} / 1254748700$.

[5] C T Chong, S Lempp, Y Yang, On the role of the collection principle for $\Sigma_{2}^{0}$-formulas in second-order reverse mathematics, Proc. Amer. Math. Soc. 138 (2010), 1093-1100; doi: 10.1090/S0002-9939-09-10115-6.

[6] C T Chong, T A Slaman, Y Yang, The metamathematics of stable Ramsey's Theorem for pairs, preprint (2012); available at http://www.math.nus.edu.sg/ $\sim$ chongct/paper-ams.pdf.

[7] D Fremlin, The generalized Banach contraction theorem (2002); available at http://www.essex.ac.uk/maths/people/fremlin/n02k16.ps.

[8] H Friedman, S G Simpson, X Yu, Periodic points and subsystems of secondorder arithmetic, Ann. Pure Appl. Logic 62 (1993), 51-64, Logic Colloquium '89 (Berlin); doi: 10.1016/0168-0072(93)90187-I.

[9] H Furstenberg, Recurrence in ergodic theory and combinatorial number theory, M. B. Porter Lectures, Princeton University Press, Princeton, N.J., 1981.

[10] K Gödel, Über eine bisher noch nicht benützte Erweiterung des finiten Standpunktes, Dialectica 12 (1958), 280-287; doi: 10.1111/j.1746-8361.1958.tb01464.x.

[11] D R Hirschfeldt, R A Shore, Combinatorial principles weaker than Ramsey's theorem for pairs, J. Symbolic Logic 72 (2007), 171-206; doi: $10.2178 / \mathrm{jsl} / 1174668391$.

[12] J R Jachymski, B Schroder, J D Stein, Jr, A connection between fixed-point theorems and tiling problems, J. Combin. Theory Ser. A 87 (1999), 273-286; doi: 10.1006/jcta.1998.2960. 
[13] J R Jachymski, J D Stein, Jr, A minimum condition and some related fixed-point theorems, J. Austral. Math. Soc. Ser. A 66 (1999) 224-243; doi: 10.1017/S144678870003932X.

[14] S C Kleene, Recursive functionals and quantifiers of finite types. I, Trans. Amer. Math. Soc. 91 (1959), 1-52; doi: 10.1090/S0002-9947-1959-0102480-9.

[15] U Kohlenbach, Higher order reverse mathematics, in: Reverse Mathematics 2001, Lect. Notes Log. 21, Assoc. Symbol. Logic, La Jolla, CA (2005), 281-295.

[16] U Kohlenbach, Applied Proof Theory: Proof Interpretations and their Use in Mathematics, Springer Monographs in Mathematics, Springer Verlag, 2008; doi: 10.1007/978-3-540-77533-1.

[17] A P Kreuzer, U Kohlenbach, Term extraction and Ramsey's theorem for pairs, J. Symbolic Logic 77 (2012), 853-895.

[18] J Merryfield, B Rothschild, J D Stein, Jr, An application of Ramsey's theorem to the Banach contraction principle, Proc. Amer. Math. Soc. 130 (2002), 927-933; doi: 10.1090/S0002-9939-01-06169-X.

[19] J Merryfield, J D Stein, Jr, A generalization of the Banach contraction principle, J. Math. Anal. Appl. 273 (2002), 112-120; doi: 10.1016/S0022247X(02)00215-9.

[20] A Montalbán, Open questions in reverse mathematics, Bull. Symbolic Logic 17 (2011), 431-454; doi: 10.2178/bsl/1309952320.

[21] C Parsons, On n-quantifier induction, J. Symbolic Logic 37 (1972), 466-482; doi: 10.1090/S0002-9947-1959-0102480-9.

[22] D Seetapun, T A Slaman, On the strength of Ramsey's theorem, Notre Dame J. Formal Logic 36 (1995), 570-582, Special Issue: Models of Arithmetic; doi: $10.1305 / \mathrm{ndjH} / 1040136917$.

[23] SG Simpson, Subsystems of Second Order Arithmetic, second edition, Perspectives in Logic, Cambridge University Press, 2009; doi: 10.1017/CBO9780511581007.

ENS Lyon, Université de Lyon, LIP (UMR 5668 CNRS ENS Lyon UCBL INRIA), 46 Allée d'Italie, 69364 Lyon Cedex 07, France

alexander . kreuzer@ens-lyon.fr

http://perso.ens-lyon.fr/alexander.kreuzer/

Received: 25 June 2012 Revised: 17 October 2012 